Document downloaded from:

http://hdl.handle.net/10251/64689

This paper must be cited as:

Cordero Barbero, A.; Ezquerro, JA.; Hernández Verón, MA.; Torregrosa Sánchez, JR. (2015). On the local convergence of a fifth-order iterative method in Banach spaces. Applied Mathematics and Computation. 251:396-403. doi:10.1016/j.amc.2014.11.084.

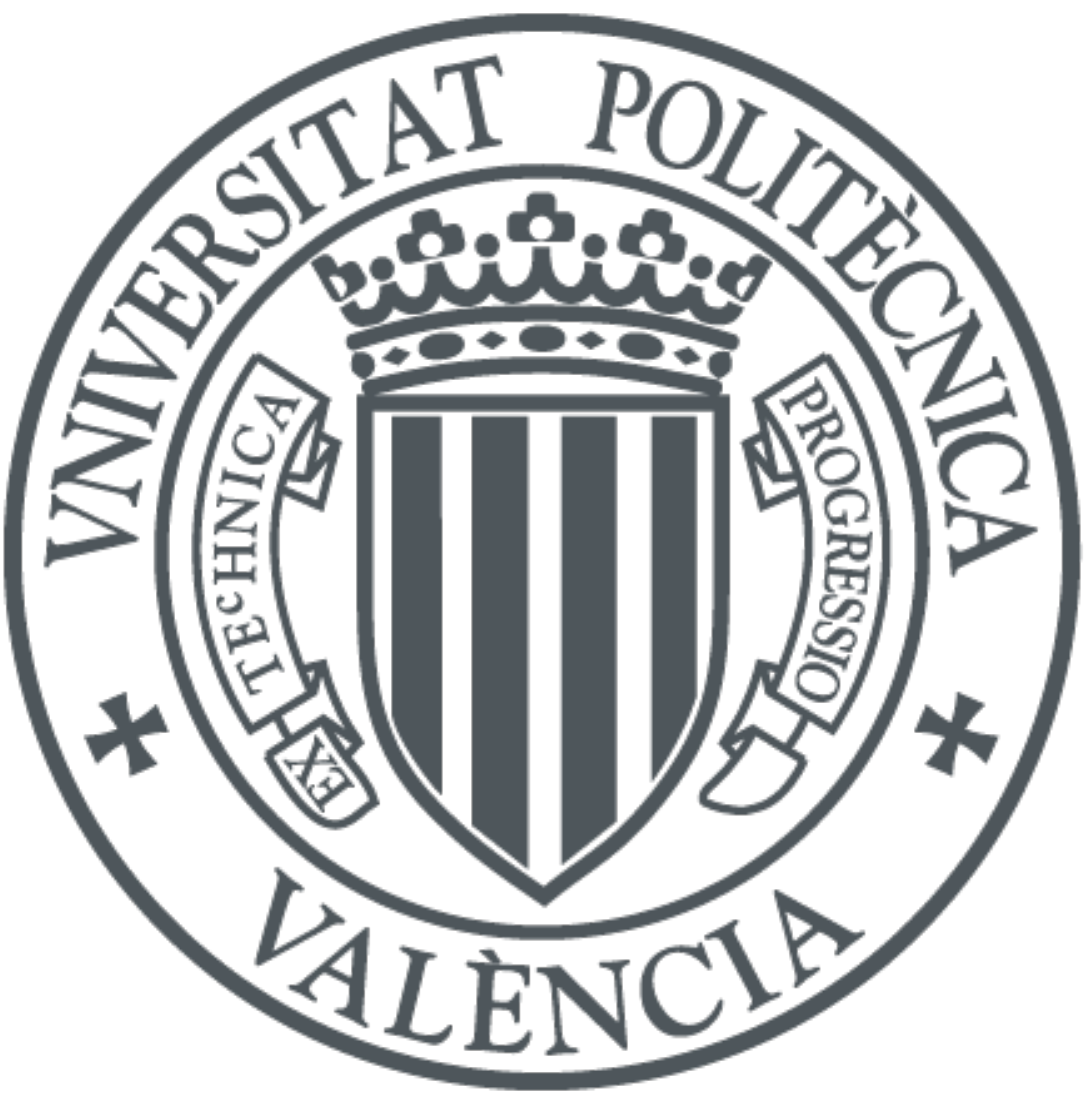

The final publication is available at

https://dx.doi.org/10.1016/j.amc.2014.11.084

Copyright Elsevier

Additional Information 


\title{
On the local convergence of a fifth-order iterative method in Banach spaces *
}

\author{
A. Cordero $^{\text {a }}$, J. A. Ezquerro ${ }^{\text {b }}$, M. A. Hernández-Verón ${ }^{b}$, J. R. Torregrosa ${ }^{\text {a }}$ \\ ${ }^{a}$ Multidisciplinary Institute of Mathematics, Universitat Politècnica de València, 46022 Valencia, Spain. \\ E-mail address: <acordero><jrtorre>@mat.upv.es \\ b Dept. of Mathematics and Computation, University of La Rioja, 26004 Logroño, Spain. \\ E-mail address: <jezquer ><mahernan>@unirioja.es
}

\begin{abstract}
A new predictor-corrector iterative procedure, that combines Newton's method as predictor scheme and a fifth-order iterative method as a corrector, is designed for solving nonlinear equations in Banach spaces. We analyze the local order of convergence and the regions of accessibility of the new method comparing it with Newton's method, both theoretical and numerically.
\end{abstract}

Keywords: Nonlinear equations, iterative method, Newton's scheme, predictor-corrector method, local convergence.

2000 Mathematics Subject Classification: 47H99, 65J15.

\section{Introduction}

Many scientific and engineering problems can be brought in the form of a nonlinear equation

$$
F(x)=0,
$$

where $F$ is a nonlinear operator defined on a non-empty open convex subset $\Omega$ of a Banach space $X$ with values in a Banach space $Y$. Problems where scalar equations, systems of equations, differential equations, integral equations, etc, can be formulated in terms of finding roots of equations of type (1). In general, the roots cannot be expressed in a closed form and this problem is commonly carried out applying iterative methods. So, starting from one or several initial approximations of a solution $x^{*}$ of equation (1), a sequence $\left\{x_{n}\right\}$ of approximations is constructed so that it converges to $x^{*}$. We can get the sequence $\left\{x_{n}\right\}$ of different ways, depending on the iterative method that is applied.

The best-known iterative scheme is Newton's method,

$$
x_{0} \text { given in } \Omega, \quad x_{n+1}=x_{n}-\left[F^{\prime}\left(x_{n}\right)\right]^{-1} F\left(x_{n}\right), n \geq 0 .
$$

${ }^{*}$ This work was supported in part by the project MTM2011-28636-C02-\{01,02\} of the Spanish Ministry of Science and Innovation. 
Observe that we need the operator $F$ to be differentiable Fréchet in order to apply Newton's method. This is one of the most used iterative method because of its good computational efficiency and accessibility to the solution $x^{*}$ of equation (1).

There are two known indices that analyze the efficiency of an iterative method. The classic efficiency index $I=p^{\frac{1}{d}}$, defined by Ostrowski in [9], provides a balance between the order of convergence $p$ and the number of functional evaluations $d$. If (1) represents a system of $n$ nonlinear equations, then, for one evaluation of $F, n$ functional evaluations are required, meanwhile the evaluation of the associated jacobian matrix $F^{\prime}$ requires $n^{2}$ functional evaluations, so that the evaluations of $F$ and $F^{\prime}$ cannot be considered in the same way. Consequently, in this case, the computational efficiency index $([10])$ is usually used, which is defined as the order of convergence to the inverse power of the operational cost, where the operational cost is the number of the operations involved in the application of the iterative method.

The efficiency is generally the most important aspect that has been taken into account when choosing an iterative method to approximate a solution of an equation (see [2, 5, 6, 7, 8]), forgetting usually the accessibility of the iterative method, which shows the domain of starting points from which the iterative method converges to the solution of the equation. This fact has led to construction of iterative methods with high efficiency, but difficult to apply in practice, since the location of starting approximations, from which the iterative methods converge to a solution of the equation, is a difficult problem to solve.

In this work, we extend to Banach spaces the M5 iterative method studied for finitedimensional problems in [1]. The M5 method has higher classic efficiency and computational efficiency indices than Newton's method (see [1]), but it presents problems of accessibility to the solution $x^{*}$ of equation (1), as we can see in Section 2. In Section 3, we prove that the local order of convergence of the M5 method is five in Banach spaces. Next, in Section 4 . taking into account that a procedure to know the accessibility of an iterative method to $x^{*}$ is to estimate the ball of convergence of the method from a local convergence result, we obtain a local convergence result for the M5 method in Banach spaces. Note that the radius of the ball of convergence, which is centered in $x^{*}$, shows the largest domain of approximations close to $x^{*}$ that guarantees the convergence of the iterative method when it starts at them. From the local convergence result, we observe that the size of the ball of convergence of the M5 method is limited with respect to the ball of convergence of Newton's method. We solve this problem in Section 5 by constructing a predictor-corrector iterative method, where the predictor scheme is Newton's one and the corrector is M5 method. This procedure allows to have the accessibility region of Newton's method and the efficiency of the M5 method. Finally, in the last section, a numerical test confirms the theoretical results obtained.

Throughout the paper we denote $\overline{B(x, \varrho)}=\{y \in X ;\|y-x\| \leq \varrho\}$ and $B(x, \varrho)=\{y \in$ $X ;\|y-x\|<\varrho\}$.

\section{Statement of the problem}

In [1], the authors propose the M5 iterative method,

$$
\left\{\begin{array}{l}
x_{0} \text { given in } \Omega, \\
y_{n}=x_{n}-\left[F^{\prime}\left(x_{n}\right)\right]^{-1} F\left(x_{n}\right), \quad n \geq 0, \\
z_{n}=y_{n}-5\left[F^{\prime}\left(x_{n}\right)\right]^{-1} F\left(y_{n}\right), \\
x_{n+1}=z_{n}-\frac{1}{5}\left[F^{\prime}\left(x_{n}\right)\right]^{-1}\left(-16 F\left(y_{n}\right)+F\left(z_{n}\right)\right),
\end{array}\right.
$$


for solving systems of nonlinear equations, and prove that its $R$-order of convergence is at least five. Moreover, from the operational cost needed to apply Newton's and M5 methods for solving systems of equations, the authors prove that the computational efficiency and the classic efficiency indices of the M5 method are higher than those of Newton's method.

As we have written in the Introduction, we must not think only of the efficiency to choose the M5 method instead of Newton's method for approximating the solution $x^{*}$ of equation (1). We must also analyze the accessibleness of both methods to $x^{*}$. We can observe this experimentally by means of the attraction basins of the iterative methods. The attraction basin of an iterative method is the set of all starting points from which the iterative method converges to $x^{*}$, once a tolerance and a maximum number of iterations is fixed.

In Figures 1 and 2, we show the attraction basins associated with the two solutions, $z^{*}=$ $\arctan (1 /(2 \sqrt{2}))=0.33983 \ldots$ and $z^{* *}=\pi-\arctan (1 /(2 \sqrt{2}))=2.80176 \ldots$, of the complex equation $F(z)=\sin z-\frac{1}{3}=0$, where $F: \mathbb{C} \longrightarrow \mathbb{C}$, when they are approximated respectively by Newton's and M5 methods. To do this, we take a rectangle $D \subset \mathbb{C}$ to represent the regions such that iterations start at every $z_{0} \in D$. In every case, a grid of $512 \times 512$ points in $D$ is considered and these points are chosen as $z_{0}$. We use the rectangle $[0,3] \times[-2.5,2.5]$ which contains the two solutions. The chosen iterative method, starting in $z_{0} \in D$, can converge to any solution or diverge. In all the examples, the tolerance $10^{-3}$ and the maximum of 25 iterations are used. We do not continue if the required tolerance is not obtained with 25 iterations and we then decide that the iterative method does not converge to any solution starting from $z_{0}$. The pictures of the attraction basins are painted using the following strategy. A colour is assigned to each attraction basin according to the root at which an iterative method converges starting from $z_{0}$. The colour is made lighter or darker according to the number of iterations needed to reach the root with fixed precision. In particular, cyan and magenta are assigned respectively for the solutions $z^{*}$ and $z^{* *}$. Finally, black is assigned if the method does not converge to any solution with a fixed tolerance and a maximum number of iterations. The graphics have been generated with Mathematica 5.1 [12]. For other strategies, reference [11] can be consulted and the references therein given.

From Figures 1 and 2, we observe that the M5 method is much more demanding with respect to the starting approximations than Newton's method, see the black colour. This clearly justifies that the M5 method is less used than Newton's method to approximate solutions of equations.

We can do a more rigorous analysis of the accessibility of an iterative method to the solution $x^{*}$ of equation (1) from a local (or semilocal) convergence result of the iterative method. From the local (or semilocal) convergence conditions, we can compare the regions of accessibility of the two methods. The region of accessibility of an iterative method provides the domain of starting points from which we have guaranteed the convergence of the iterative method. In other words, the region of accessibility represents the domain of starting points that satisfies the convergence conditions required to the iterative method.

In this paper, we fix the region of accessibility of Newton's method from the local convergence result given by Dennis and Schnabel in [4], which is a classic and known convergence result. Next, we obtain the region of accessibility of the M5 method from the same conditions as those given by Dennis and Schnabel for Newton's method and we see that it is smaller than that of Newton's one. After that, we construct a predictor-corrector iterative method, where Newton's method is the predictor and M5 is the corrector, with higher efficiency than that of Newton's method, but with the same region of accessibility. In consequence, the new iterative method will be more appropriate than Newton's method for approximating the solution $x^{*}$ of equation (1). 


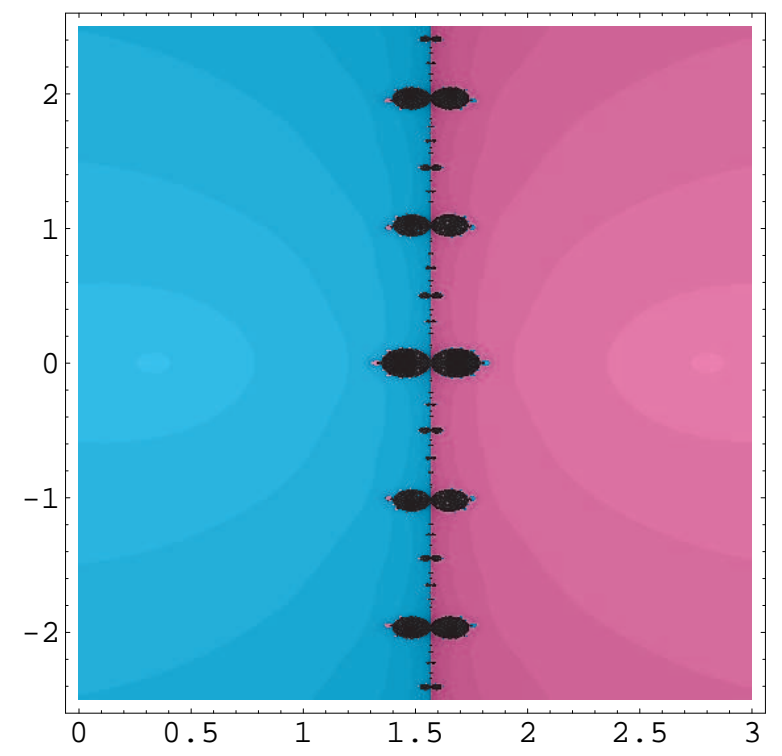

Figure 1: Attraction basins of Newton's method when it is used to approximate the two solutions of the equation $F(z)=\sin z-$ $\frac{1}{3}=0$.

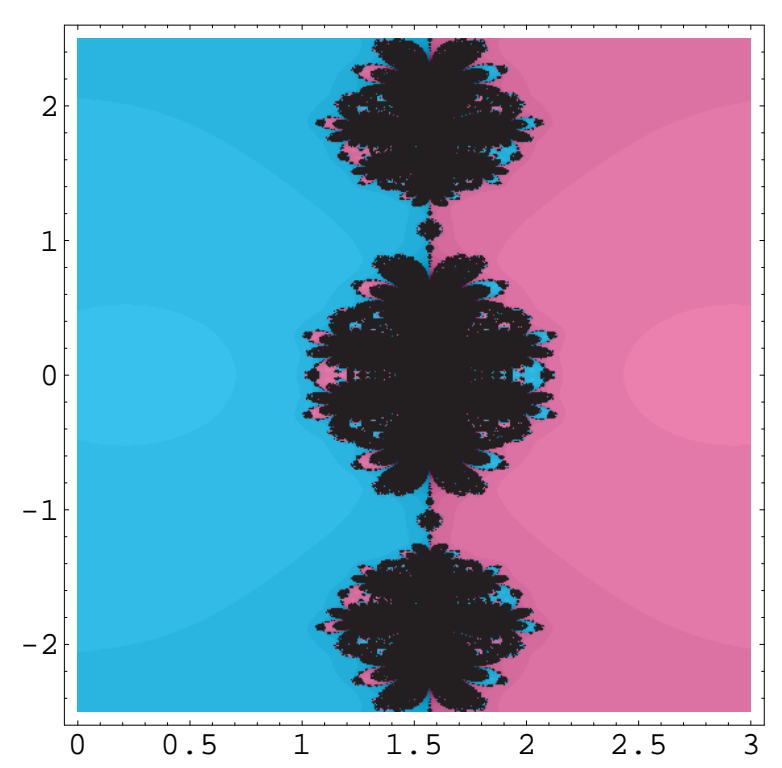

Figure 2: Attraction basins of the M5 method when it is used to approximate the two solutions of the equation $F(z)=\sin z-$ $\frac{1}{3}=0$.

\section{Local order of convergence of the M5 method}

In the following, we remember some known notions and results that we need in order to study the local order of convergence of the M5 method (see [3]).

Let $F: \Omega \subseteq X \longrightarrow Y$ be a sufficiently differentiable operator in $\Omega$. The $q$-th derivative of $F$ at $u \in \Omega, q \geq 1$, is the $q$-linear function $F^{(q)}(u): \Omega \times \cdots \times \Omega \longrightarrow Y$ such that $F^{(q)}(u)\left(v_{1}, \ldots, v_{q}\right) \in Y$. It is easy to observe that

1. $F^{(q)}(u)\left(v_{1}, \ldots, v_{q-1}, \cdot\right) \in \mathcal{L}(\Omega, Y)$, where $\mathcal{L}(\Omega, Y)$ is the set of the bounded linear operators from $\Omega$ into $Y$.

2. $F^{(q)}(u)\left(v_{\sigma(1)}, \ldots, v_{\sigma(q)}\right)=F^{(q)}(u)\left(v_{1}, \ldots, v_{q}\right)$, for all permutation $\sigma$ of $\{1,2, \ldots, q\}$.

After this, we use the following notation:

(a) $F^{(q)}(u)\left(v_{1}, \ldots, v_{q}\right)=F^{(q)}(u) v_{1} \cdots v_{q}$,

(b) $F^{(q)}(u) v^{q-1} F^{(p)} v^{p}=F^{(q)}(u) F^{(p)}(u) v^{q+p-1}$.

On the other hand, for $x^{*}+h \in \Omega$ lying in a neighborhood of a solution $x^{*}$ of $F(x)=0$, we can apply Taylor's expansion and assume that $F^{\prime}\left(x^{*}\right) \neq 0$, we have

$$
F\left(x^{*}+h\right)=F^{\prime}\left(x^{*}\right)\left[h+\sum_{q=2}^{p-1} c_{q} h^{q}\right]+O\left(h^{p}\right),
$$

where $c_{q}=\frac{1}{q !}\left[F^{\prime}\left(x^{*}\right)\right]^{-1} F^{(q)}\left(x^{*}\right), q \geq 2$. We observe that $c_{q} h^{q} \in \Omega$, since $F^{(q)}\left(x^{*}\right) \in \mathcal{L}(\Omega \times \cdots \times$ $\Omega, Y)$ and $\left[F^{\prime}\left(x^{*}\right)\right]^{-1} \in \mathcal{L}(Y, \Omega)$.

In addition, we can express $F^{\prime}$ as

$$
F^{\prime}\left(x^{*}+h\right)=F^{\prime}\left(x^{*}\right)\left[I+\sum_{q=2}^{p-1} q c_{q} h^{q-1}\right]+O\left(h^{p}\right),
$$


where $I$ is the identity matrix. Therefore, $q c_{q} h^{q-1} \in \mathcal{L}(\Omega, Y)$. From (3), we assume

$$
\left[F^{\prime}\left(x^{*}+h\right)\right]^{-1}=\left[I+X_{2} h+X_{3} h^{2}+X_{4} h^{4}+\cdots\right]\left[F^{\prime}\left(x^{*}\right)\right]^{-1}+O\left(h^{p}\right)
$$

and, taking into account that $\left[F^{\prime}\left(x^{*}+h\right)\right]^{-1} F^{\prime}\left(x^{*}+h\right)=F^{\prime}\left(x^{*}+h\right)\left[F^{\prime}\left(x^{*}+h\right)\right]^{-1}=I$,

$$
X_{s}=-\sum_{j=2}^{s} j X_{s-j+1} c_{j}, \quad s=2,3, \ldots,
$$

where $X_{1}=I$.

If we denote $e_{n}=x_{n}-x^{*}$ the error in the $n$th iteration, the equation

$$
e_{n+1}=M e_{n}^{p}+O\left(e_{n}^{p+1}\right),
$$

where $M$ is a $p$-linear function, $M \in \mathcal{L}(\Omega \times \cdots \times \Omega, Y)$, is called the error equation and $p$ is the order of convergence. Notice that $e_{n}^{p}$ is $\left(e_{n}, e_{n}, \ldots, e_{n}\right)$.

In the next result, we prove the local order of convergence of the M5 method, by using the Taylor expansions, and obtain the error equation.

Theorem 1 Let $F: \Omega \subseteq X \rightarrow Y$ be a nonlinear continuously differentiable enough operator on a non-empty open convex domain $\Omega$ of a Banach space $X$ with values in a Banach space $Y$ and $x^{*} \in \Omega$ a solution of the equation $F(x)=0$. Suppose that $F^{\prime}(x)$ is continuous and nonsingular at $x^{*}$. Then, the sequence $\left\{x_{n}\right\}$, given by the M5 method, converges to $x^{*}$ with local order of convergence five. Moreover, the error equation is

$$
e_{n+1}=\left(-2 c_{2} c_{3} c_{2}+6 c_{3} c_{2}^{2}+14 c_{2}^{4}\right) e_{n}^{5}+O\left(e_{n}^{6}\right),
$$

where $c_{j}=\frac{1}{j !}\left[F^{\prime}\left(x^{*}\right)\right]^{-1} F^{(j)}\left(x^{*}\right)$, for $j=2,3, \ldots$, and $e_{n}=x_{n}-x^{*}$.

Proof. We expand $F\left(x_{n}\right)$ and $F^{\prime}\left(x_{n}\right)$ in the Taylor series around the solution $x^{*}$,

$$
\begin{gathered}
F\left(x_{n}\right)=F^{\prime}\left(x^{*}\right)\left[e_{n}+c_{2} e_{n}^{2}+c_{3} e_{n}^{3}+c_{4} e_{n}^{4}+c_{5} e_{n}^{5}\right]+O\left(e_{n}^{6}\right), \\
F^{\prime}\left(x_{n}\right)=F^{\prime}\left(x^{*}\right)\left[I+2 c_{2} e_{n}+3 c_{3} e_{n}^{2}+4 c_{4} e_{n}^{3}+5 c_{5} e_{n}^{4}\right]+O\left(e_{n}^{5}\right) .
\end{gathered}
$$

Suppose that the Taylor expansion of the inverse operator at $x_{n}$ is

$$
F^{\prime}\left(x_{n}\right)^{-1}=\left[I+X_{2} e_{n}+X_{3} e_{n}^{2}+X_{4} e_{n}^{3}+X_{5} e_{n}^{4}\right] F^{\prime}\left(x^{*}\right)^{-1}+O\left(e_{n}^{5}\right) .
$$

From $\left[F^{\prime}\left(x_{n}\right)\right]^{-1} F^{\prime}\left(x_{n}\right)=F^{\prime}\left(x_{n}\right)\left[F^{\prime}\left(x_{n}\right)\right]^{-1}=I$, we obtain

$$
\begin{aligned}
& X_{2}=-2 c_{2}, \\
& X_{3}=4 c_{2}^{2}-3 c_{3}, \\
& X_{4}=6 c_{3} c_{2}-8 c_{2}^{3}+6 c_{2} c_{3}-4 c_{4}, \\
& X_{5}=16 c_{2}^{4}-12 c_{3} c_{2}^{2}-12 c_{2} c_{3} c_{2}+8 c_{4} c_{2}+9 c_{3}^{2}-12 c_{2}^{2} c_{3}+8 c_{2} c_{4}-5 c_{5} .
\end{aligned}
$$

Then, the error at the first step of the process can be expressed as

$$
\begin{aligned}
y_{n}-x^{*}= & e_{n}-\left[e_{n}+c_{2} e_{n}^{2}+c_{3} e_{n}^{3}+c_{4} e_{n}^{4}+c_{5} e_{n}^{5}+X_{2} e_{n}^{2}+X_{2} c_{2} e_{n}^{3}+X_{2} c_{3} e_{n}^{4}+X_{2} c_{4} e_{n}^{5}\right. \\
& \left.+X_{3} e_{n}^{3}+X_{3} c_{2} e_{n}^{4}+X_{3} c_{3} e_{n}^{5}+X_{4} e_{n}^{4}+X_{4} c_{2} e_{n}^{5}+X_{5} e_{n}^{5}\right]+O\left(e_{n}^{6}\right) \\
= & c_{2} e_{n}^{2}+2\left(c_{3}-c_{2}^{2}\right) e_{n}^{3}+\left(4 c_{2}^{3}-4 c_{2} c_{3}-3 c_{3} c_{2}+3 c_{4}\right) e_{n}^{4} \\
& +\left(4 c_{5}-6 c_{2} c_{4}+8 c_{2}^{2} c_{3}-6 c_{3}^{2}-4 c_{4} c_{2}+6 c_{2} c_{3} c_{2}+6 c_{3} c_{2}^{2}-8 c_{2}^{4}\right) e_{n}^{5}+O\left(e_{n}^{6}\right) .
\end{aligned}
$$


Then, as $F\left(y_{n}\right)=F^{\prime}\left(x^{*}\right)\left[y_{n}-x^{*}+c_{2}\left(y_{n}-x^{*}\right)^{2}+c_{3}\left(y_{n}-x^{*}\right)^{3}\right]+O\left(\left(y_{n}-x^{*}\right)^{4}\right), F\left(y_{n}\right)$ can be expressed in terms of the error at $x_{n}$ as

$$
\begin{aligned}
F\left(y_{n}\right)= & F^{\prime}\left(x^{*}\right)\left[c_{2} e_{n}^{2}+2\left(c_{3}-c_{2}^{2}\right) e_{n}^{3}+\left(5 c_{2}^{3}-4 c_{2} c_{3}-3 c_{3} c_{2}+3 c_{4}\right) e_{n}^{4}\right. \\
& \left.+\left(4 c_{5}-6 c_{2} c_{4}+10 c_{2}^{2} c_{3}-6 c_{3}^{2}-4 c_{4} c_{2}+8 c_{2} c_{3} c_{2}+6 c_{3} c_{2}^{2}-12 c_{2}^{4}\right) e_{n}^{5}\right]+O\left(e_{n}^{6}\right) .
\end{aligned}
$$

Moreover, the expansion of the inverse operator on $F\left(y_{n}\right)$ is

$$
\begin{aligned}
{\left[F^{\prime}\left(x_{n}\right)\right]^{-1} F\left(y_{n}\right)=} & c_{2} e_{n}^{2}+2\left(c_{3}-2 c_{2}^{2}\right) e_{n}^{3}+\left(13 c_{2}^{3}-8 c_{2} c_{3}-6 c_{3} c_{2}+3 c_{4}\right) e_{n}^{4} \\
& +\left(4 c_{5}-12 c_{2} c_{4}+26 c_{2}^{2} c_{3}-12 c_{3}^{2}-8 c_{4} c_{2}+20 c_{2} c_{3} c_{2}+18 c_{3} c_{2}^{2}-38 c_{2}^{4}\right) e_{n}^{5}+O\left(e_{n}^{6}\right)
\end{aligned}
$$

and the expansion of the error at the second step is

$$
\begin{aligned}
z_{n}-x^{*}= & y_{n}-x^{*}-5\left[F^{\prime}\left(x_{n}\right)\right]^{-1} F\left(y_{n}\right) \\
= & -4 c_{2} e_{n}^{2}+\left(18 c_{2}^{2}-8 c_{3}\right) e_{n}^{3}+\left(-61 c_{2}^{3}+36 c_{2} c_{3}+27 c_{3} c_{2}-12 c_{4}\right) e_{n}^{4} \\
& +\left(-16 c_{5}+54 c_{2} c_{4}-122 c_{2}^{2} c_{3}+54 c_{3}^{2}+36 c_{4} c_{2}-94 c_{2} c_{3} c_{2}-84 c_{3} c_{2}^{2}+182 c_{2}^{4}\right) e_{n}^{5}+O\left(e_{n}^{6}\right) .
\end{aligned}
$$

Then,

$$
\begin{aligned}
F\left(z_{n}\right)= & F^{\prime}\left(x^{*}\right)\left[z_{n}-x^{*}+c_{2}\left(z_{n}-x^{*}\right)^{2}+c_{3}\left(z_{n}-x^{*}\right)^{3}\right]+O\left(\left(z_{n}-x^{*}\right)^{4}\right) \\
= & F^{\prime}\left(x^{*}\right)\left[-4 c_{2} e_{n}^{2}+\left(18 c_{2}^{2}-8 c_{3}\right) e_{n}^{3}+\left(-45 c_{2}^{3}+36 c_{2} c_{3}+27 c_{3} c_{2}-12 c_{4}\right) e_{n}^{4}\right. \\
& \left.+\left(-16 c_{5}+54 c_{2} c_{4}-90 c_{2}^{2} c_{3}+54 c_{3}^{2}+36 c_{4} c_{2}-62 c_{2} c_{3} c_{2}-84 c_{3} c_{2}^{2}+38 c_{2}^{4}\right) e_{n}^{5}\right]+O\left(e_{n}^{6}\right)
\end{aligned}
$$

and

$$
\begin{aligned}
& {\left[F^{\prime}\left(x_{n}\right)\right]^{-1}\left(F\left(z_{n}\right)-16 F\left(y_{n}\right)\right)=} \\
& \quad-20 c_{2} e_{n}^{2}+\left(90 c_{2}^{2}-40 c_{3}\right) e_{n}^{3}+\left(-305 c_{2}^{3}+180 c_{2} c_{3}+135 c_{3} c_{2}-60 c_{4}\right) e_{n}^{4} \\
& \quad+\left(-80 c_{5}+270 c_{2} c_{4}-610 c_{2}^{2} c_{3}+270 c_{3}^{2}+180 c_{4} c_{2}-460 c_{2} c_{3} c_{2}-450 c_{3} c_{2}^{2}+840 c_{2}^{4}\right) e_{n}^{5}+O\left(e_{n}^{6}\right) .
\end{aligned}
$$

Finally, the error equation of the method is

$$
\begin{aligned}
x_{n+1}-x^{*} & =z_{n}-x^{*}-\frac{1}{5}\left[F^{\prime}\left(x_{n}\right)\right]^{-1}\left(F\left(z_{n}\right)-16 F\left(y_{n}\right)\right) \\
& =\left(-2 c_{2} c_{3} c_{2}+6 c_{3} c_{2}^{2}+14 c_{2}^{4}\right) e_{n}^{5}+O\left(e_{n}^{6}\right),
\end{aligned}
$$

and then, the local order of the M5 method is five.

\section{Regions of accessibility of Newton's method and the M5 method}

The local convergence results for iterative methods require conditions on the operator $F$ and the solution $x^{*}$ of equation (1). Note that a local result provides what we call ball of convergence and denote by $B\left(x^{*}, \varepsilon\right)$. From the value $\varepsilon$, the ball of convergence gives information about the accessibility of the solution $x^{*}$.

An interesting local result for Newton's method is given in [4] by Dennis and Schnabel, where the following conditions are required:

$\left(L_{1}\right)$ Let $x^{*}$ be a solution of equation (1) and exist $r>0$, so that $B\left(x^{*}, r\right) \subset \Omega$ and the operator $\left[F^{\prime}\left(x^{*}\right)\right]^{-1}$ exists with $\left\|\left[F^{\prime}\left(x^{*}\right)\right]^{-1}\right\| \leq \gamma$ and $\gamma>0$, 
$\left(L_{2}\right)\left\|F^{\prime}(x)-F^{\prime}(y)\right\| \leq K\|x-y\|, K \geq 0$, for all $x, y \in \Omega$.

Dennis and Schnabel prove, under conditions $\left(L_{1}\right)$ and $\left(L_{2}\right)$, that, for any starting point belonging to $B\left(x^{*}, \varepsilon\right)$, where $\varepsilon=\min \{r, R\}$ and $R=\frac{1}{2 \gamma K}$, Newton's method is convergent.

In the following, we establish a local convergence result for the M5 method under conditions $\left(L_{1}\right)$ and $\left(L_{2}\right)$.

Theorem 2 Let $F: \Omega \subseteq X \rightarrow Y$ be a nonlinear continuously differentiable operator on a non-empty open convex domain $\Omega$ of a Banach space $X$ with values in a Banach space $Y$. Suppose that conditions $\left(L_{1}\right)$ and $\left(L_{2}\right)$ are satisfied. Then, there exists $\tilde{\varepsilon}>0$, such that the sequence $\left\{x_{n}\right\}$, given by the M5 method, is well-defined and converges to a solution $x^{*}$ of the equation $F(x)=0$ from every point $x_{0} \in B\left(x^{*}, \tilde{\varepsilon}\right)$.

Proof. Let $\tilde{\varepsilon}=\min \{r, \widetilde{R}\}$, where $\widetilde{R}=\frac{13-3 \sqrt{5}}{31 \gamma K}$. First, we prove, for all $x \in B\left(x^{*}, \tilde{\varepsilon}\right)$, that the operator $\left[F^{\prime}(x)\right]^{-1}$ exists and $\left\|\left[F^{\prime}(x)\right]^{-1}\right\| \leq \frac{\gamma}{1-\gamma K \tilde{\varepsilon}}$. For this, we consider

$$
\left\|I-\left[F^{\prime}\left(x^{*}\right)\right]^{-1} F^{\prime}(x)\right\| \leq\left\|\left[F^{\prime}\left(x^{*}\right)\right]^{-1}\right\|\left\|F^{\prime}\left(x^{*}\right)-F^{\prime}(x)\right\| \leq \gamma K\left\|x-x^{*}\right\|<\gamma K \tilde{\varepsilon}<1 .
$$

Now, by the Banach's lemma on invertible operators, the operator $\left[F^{\prime}(x)\right]^{-1}$ exists and $\left\|\left[F^{\prime}(x)\right]^{-1}\right\|<$ $\frac{1}{1-\gamma K \tilde{\varepsilon}}\left\|\left[F^{\prime}\left(x^{*}\right)\right]^{-1}\right\| \leq \frac{\gamma}{1-\gamma K \tilde{\varepsilon}}$.

As $x_{0} \in B\left(x^{*}, \tilde{\varepsilon}\right)$, then the operator $\Gamma_{0}=\left[F^{\prime}\left(x_{0}\right)\right]^{-1}$ exists, $\left\|\Gamma_{0}\right\|<\frac{\gamma}{1-\gamma K \tilde{\varepsilon}}, y_{0}$ is well-defined and

$$
y_{0}-x^{*}=x_{0}-\Gamma_{0} F\left(x_{0}\right)-x^{*}=\Gamma_{0} \int_{0}^{1}\left(F^{\prime}\left(x^{*}+s\left(x^{*}-x_{0}\right)\right)-F^{\prime}\left(x_{0}\right)\right) d s\left(x^{*}-x_{0}\right) .
$$

Then, $y_{0} \in B\left(x^{*}, \tilde{\varepsilon}\right)$, since $\left\|y_{0}-x^{*}\right\|<\frac{\delta}{2}\left\|x_{0}-x^{*}\right\|<\left\|x_{0}-x^{*}\right\|$, where $\delta=\frac{\gamma K \tilde{\varepsilon}}{1-\gamma K \tilde{\varepsilon}}$.

Moreover, $z_{0}$ is well-defined and $z_{0} \in B\left(x^{*}, \tilde{\varepsilon}\right)$, since

$$
\begin{aligned}
z_{0}-x^{*} & =y_{0}-5 \Gamma_{0} F\left(y_{0}\right)-x^{*} \\
& =5 \Gamma_{0} \int_{0}^{1}\left(F^{\prime}\left(y_{0}+s\left(x^{*}-y_{0}\right)\right)-F^{\prime}\left(x_{0}\right)\right) d s\left(x^{*}-y_{0}\right)+4\left(x^{*}-y_{0}\right)
\end{aligned}
$$

and $\left\|z_{0}-x^{*}\right\|<f(\delta)\left\|x_{0}-x^{*}\right\|<\left\|x_{0}-x^{*}\right\|$, where $f(t)=t\left(\frac{5}{8} t^{2}+\frac{5}{2} t+2\right)$ and $f(\delta)<1$.

Furthermore, $x_{1}$ is well-defined and $x_{1} \in B\left(x^{*}, \tilde{\varepsilon}\right)$, since

$$
\begin{aligned}
x_{1}-x^{*}= & z_{0}-\Gamma_{0}\left(-\frac{16}{5} F\left(y_{0}\right)+\frac{1}{5} F\left(z_{0}\right)\right) \\
= & \Gamma_{0}\left(\frac{1}{5} \int_{0}^{1}\left(F^{\prime}\left(z_{0}+s\left(x^{*}-z_{0}\right)\right)-F^{\prime}\left(x_{0}\right)\right) d s\left(x^{*}-z_{0}\right)\right. \\
& \left.+\frac{4}{5} \int_{0}^{1}\left(F^{\prime}\left(y_{0}+s\left(x^{*}-y_{0}\right)\right)-F^{\prime}\left(x_{0}\right)\right) d s\left(x^{*}-y_{0}\right)\right)
\end{aligned}
$$

and $\left\|x_{1}-x^{*}\right\|<g(\delta)\left\|x_{0}-x^{*}\right\|<\left\|x_{0}-x^{*}\right\|$, where $g(t)=t f(t)\left(\frac{f(t)}{10}+1\right)+\frac{t^{2}}{5}(t+2)$ and $g(\delta)<1$.

Following now an inductive procedure on $n \in \mathbb{N}$, we have:

$$
\left\|y_{n}-x^{*}\right\|<\frac{\delta}{2}\left\|x_{n}-x^{*}\right\|, \quad\left\|z_{n}-x^{*}\right\|<f(\delta)\left\|x_{n}-x^{*}\right\|, \quad\left\|x_{n+1}-x^{*}\right\|<g(\delta)\left\|x_{n}-x^{*}\right\| .
$$


Therefore, $\left\|x_{n}-x^{*}\right\|<g(\delta)^{n}\left\|x_{0}-x^{*}\right\|$, for all $n \in \mathbb{N}$, and consequently $\lim _{n \rightarrow+\infty} x_{n}=x^{*}$.

As the radii of the balls of convergence of both methods are given by conditions $\left(L_{1}\right)$ and $\left(L_{2}\right)$, it is clear that the radius of the ball of convergence of Newton's method is significantly larger than that of the M5 method, since $\frac{1}{2 \gamma K}>\frac{13-3 \sqrt{5}}{31 \gamma K}$.

If we consider again the complex equation $F(z)=\sin z-\frac{1}{3}=0$, the balls of convergence of Newton's method and the M5 method are respectively $B(\tilde{z}, 0.17342 \ldots)$ and $B(\tilde{z}, 0.07039 \ldots)$, where $\tilde{z}=z^{*}, z^{* *}$ (see Figures 3 and 4 ). Observe the difference that exists between the two balls of convergence, which also coincides with the difference shown by the attractions basins in Figures 1 and 2.

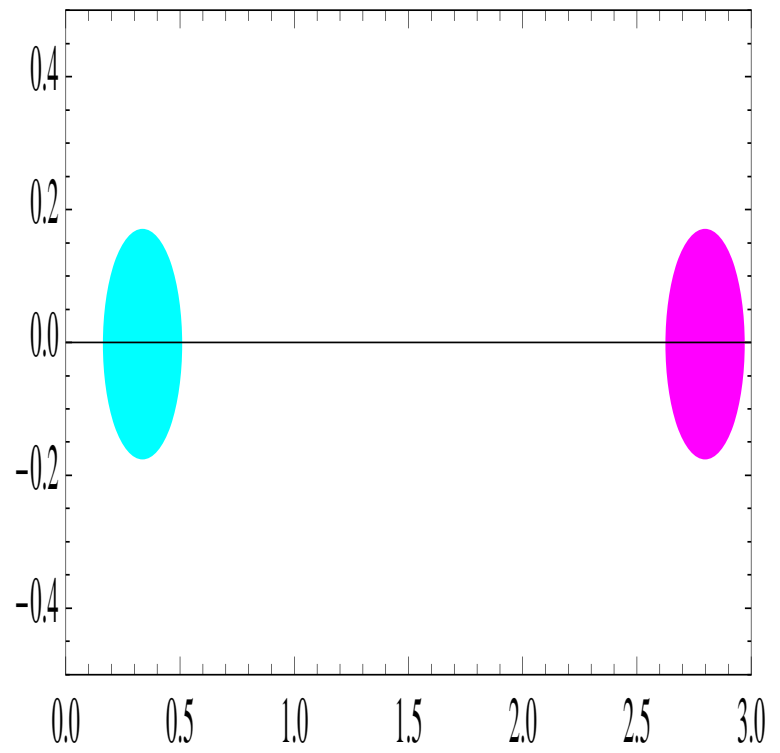

Figure 3: Balls of convergence of Newton's method when it is used to approximate the two solutions of the equation $F(z)=\sin z-$ $\frac{1}{3}=0$.

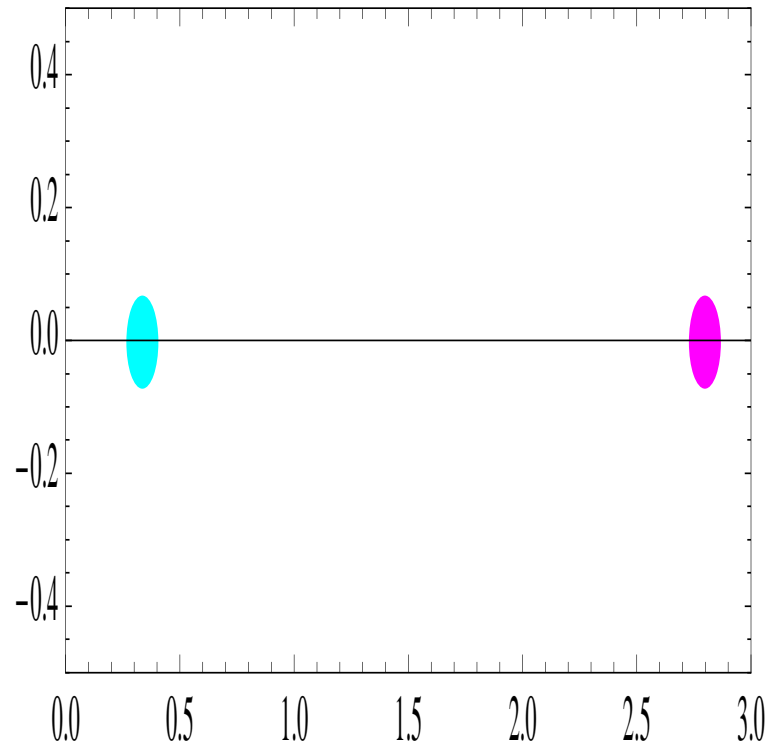

Figure 4: Balls of convergence of the M5 method when it is used to approximate the two solutions of the equation $F(z)=\sin z-$ $\frac{1}{3}=0$.

\section{A predictor-corrector iterative method}

Our next aim is to design an iterative method from the M5 method with the same accessibility as Newton's method. For this, we construct a new hybrid iterative method from the M5 method that converges when it starts from the same starting points from which Newton's method is convergent. So, we define the following iterative method:

$$
\left\{\begin{array}{l}
\left\{\begin{array}{l}
u_{0} \in B\left(x^{*}, \varepsilon\right), \text { where } \varepsilon=\min \{r, R\} \text { and } R=\frac{1}{2 \gamma K}, \\
u_{n}=u_{n-1}-\left[F^{\prime}\left(u_{n-1}\right)\right]^{-1} F\left(u_{n-1}\right), \quad n=0,1, \ldots, N_{0},
\end{array}\right. \\
\left\{\begin{array}{l}
x_{0}=u_{N_{0}}, \\
y_{k-1}=x_{k-1}-\left[F^{\prime}\left(x_{k-1}\right)\right]^{-1} F\left(x_{k-1}\right), \quad k \in \mathbb{N}, \\
z_{k-1}=y_{k-1}-5\left[F^{\prime}\left(x_{k-1}\right)\right]^{-1} F\left(y_{k-1}\right), \\
x_{k}=z_{k-1}-\frac{1}{5}\left[F^{\prime}\left(x_{k-1}\right)\right]^{-1}\left(-16 F\left(y_{k-1}\right)+F\left(z_{k-1}\right)\right),
\end{array}\right.
\end{array}\right.
$$


where $x_{0}=u_{N_{0}} \in B\left(x^{*}, \tilde{\varepsilon}\right), \tilde{\varepsilon}=\min \{r, \widetilde{R}\}$ and $\widetilde{R}=\frac{13-3 \sqrt{5}}{31 \gamma K}$, and provided that $B\left(x^{*}, r\right) \subset \Omega$. In this case, we can use Newton's method for a finite number of steps $N_{0}$ and then apply the M5 method to accelerate the convergence. To do this, we have to guarantee the existence of $N_{0}$.

Now, we study the local convergence of method (6). Since $\varepsilon=\min \{r, R\}, \tilde{\varepsilon}=\min \{r, \widetilde{R}\}$ and $\widetilde{R}<R$, then three cases can be given:

$$
\varepsilon=\tilde{\varepsilon}=r, \quad \varepsilon=r \text { and } \tilde{\varepsilon}=\widetilde{R}, \quad \varepsilon=R \text { and } \tilde{\varepsilon}=\widetilde{R} .
$$

Observe that the last two cases lead to $\tilde{\varepsilon}<\varepsilon$. In consequence, we only have to consider two cases: $\tilde{\varepsilon}=\varepsilon$ and $\tilde{\varepsilon}<\varepsilon$. But, the first one leads to the fact that the two balls of convergence are the same, so that we can apply the M5 method from the beginning without applying Newton's method for a finite number of steps $N_{0}$. Therefore, the most interesting case is when $\tilde{\varepsilon}<\varepsilon$.

From the local convergence result for Newton's method given in [4] by Dennis and Schnabel, it follows

$$
\left\|u_{n}-x^{*}\right\| \leq\left(\frac{1}{2}\right)^{n}\left\|u_{0}-x^{*}\right\|<\left(\frac{1}{2}\right)^{n} \varepsilon .
$$

Therefore, we look for the first value of $n$ such that $\left(\frac{1}{2}\right)^{n} \varepsilon<\tilde{\varepsilon}$. Taking logarithms, we find a value $N_{0} \in \mathbb{N}$ such that $x_{0}=u_{N_{0}} \in B\left(x^{*}, \tilde{\varepsilon}\right)$. So, $N_{0}>\frac{\log \left(\frac{\varepsilon}{\tilde{\varepsilon}}\right)}{\log 2}$ and, consequently,

$$
N_{0}=1+\left[\frac{\log \left(\frac{\varepsilon}{\tilde{\varepsilon}}\right)}{\log 2}\right] \text {, }
$$

where $[t]$ denotes the integer part of any real number $t$.

Finally, once the value $N_{0}$ is a priori estimated, we summarize all the above in the following result, which guarantees the local convergence of method (6).

Theorem 3 Let $F: \Omega \subseteq X \rightarrow Y$ be a nonlinear continuously differentiable operator on a non-empty open convex domain $\Omega$ of a Banach space $X$ with values in a Banach space $Y$. Suppose that conditions $\left(L_{1}\right)$ and $\left(L_{2}\right)$ are satisfied. Then, there exist $\varepsilon, \tilde{\varepsilon}>0$ such that the sequence $\left\{x_{n}\right\}$, given by method (6), with $N_{0}$ defined in (7), is well-defined and converges to a solution $x^{*}$ of the equation $F(x)=0$ from every point $x_{0} \in B\left(x^{*}, \varepsilon\right)$.

The previous local convergence result guarantees that method (6) has the same ball of convergence as Newton's method.

\section{An example}

Next, we illustrate the previous result with the following example given in [4]. We choose the max-norm.

Let $F: \mathbb{R}^{3} \rightarrow \mathbb{R}^{3}$ be defined as $F(x, y, z)=\left(x, y^{2}+y, e^{z}-1\right)$. It is obvious that $x^{*}=(0,0,0)$ is a solution of the system.

From $F$, we have

$$
F^{\prime}(x, y, z)=\left(\begin{array}{ccc}
1 & 0 & 0 \\
0 & 2 y+1 & 0 \\
0 & 0 & e^{z}
\end{array}\right)
$$

and $F^{\prime}\left(x^{*}\right)$ is the identity matrix $3 \times 3$. So, $\left\|F^{\prime}\left(x^{*}\right)^{-1}\right\|=1$ and $\gamma=1$. On the other hand, there exists $r=1$, such that $B(0, r)=\left\{w \in \mathbb{R}^{3}:\|w\|<1\right\} \subset \mathbb{R}^{3}$, and it is easy to prove that

$$
\left\|F^{\prime}(x, y, z)-F^{\prime}(u, v, w)\right\|=\max \left\{2|y-v|,\left|e^{z}-e^{w}\right|\right\} \leq 3\|(x, y, z)-(u, v, w)\|,
$$


in $B(0, r)$. Therefore, $R=\frac{1}{2 \gamma K}=\frac{1}{6}$ and Newton's method is convergent from any starting point belonging to $B\left(x^{*}, 1 / 6\right)$. However, from Theorem 2, the M5 method is convergent from any starting point belonging to $B\left(x^{*}, 0.067653 \ldots\right)$. This shows again that the accessibility of the M5 method is reduced. If we consider method (6), the accessibility region is that of Newton's method and the number of iterations needed with the predictor is $2\left(N_{0}=2\right)$. Then, the M5 method is applied and the convergence of fifth-order is guaranteed.

\section{References}

[1] V. Arroyo, A. Cordero and J.R. Torregrosa, Approximation of artificial satellites preliminary orbits: The efficiency challenge, Math. Comput. Modelling 54 (2011) 1802-1807.

[2] A. Cordero,J.L. Hueso, E. Martínez and J.R. Torregrosa, Efficient high-order methods based on golden ratio for nonlinear systems, Appl. Math. Comput. 217 (2011) 4548-4556.

[3] A. Cordero,J.L. Hueso, E. Martínez and J.R. Torregrosa, A modified Newton-Jarratt composition, Numerical Algorithms 55 (2010) 87-99.

[4] J. E. Dennis and R. B. Schnabel, Numerical methods for unconstrained optimization and nonlinear equations, SIAM, Philadelphia, 1996.

[5] J. A. Ezquerro and M. A. Hernández, New iterations of $R$-order four with reduced computational cost, BIT 49, 2 (2009) 325-342.

[6] J. A. Ezquerro, M. A. Hernández and N. Romero, Improving the efficiency index of onepoint iterative processes, J. Comput. Appl. Math. 223, 2 (2009) 879-892.

[7] M. Grau-Sánchez and J. L. Díaz-Barrero, On computational efficiency for multi-precision zero-finding methods, Appl. Math. Comput. 181 (2006) 402-412.

[8] M. Grau-Sánchez, Improvement of the efficiency of some three-step iterative like-Newton methods, Numer. Math. 107 (2007) 131-146.

[9] A.M. Ostrowski, Solutions of equations and systems of equations, Academic Press, New York-London, 1966.

[10] J.F. Traub, Iterative methods for the solution of equations, Chelsea Publishing Company, New York, 1982.

[11] J. L. Varona, Graphic and numerical comparison between iterative methods, Math. Intelligencer 24 (2002) 37-46.

[12] S. Wolfram, The Mathematica book, 5th ed., Wolfram Media, Cambridge University Press, 2003. 